\title{
The Key Factor in the Favorable Dynamics of Periodontal Conditions in Pregnant Women
}

\author{
VA Prohodnaya* and Chibichyan EH \\ Health Ministry of Russian Federation, Rostov State Medical University, Russia
}

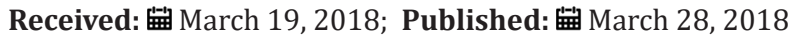

*Corresponding author: VA Prohodnaya, Health Ministry of Russian Federation, Rostov State Medical University, 344022, Rostov-on-Don, Russian Federation, Russia

\begin{abstract}
Pregnant women have one of the highest risks of dental diseases [1]. According to foreign researchers, the prevalence of periodontal pathology, ranging from gingivitis and ending with periodontitis, is 10-60\% [2]. In the work of LaineM.A.it is established that pregnant women during the gestational period suffer from gingivitis in $30-100 \%$, parodontitis - in $5-20 \%$ [3].
\end{abstract}

The aim was to study the influence of adherence of pregnant women to the treatment of periodontal diseases on the course of this pathology.

\section{Materials and Methods of Research}

398 pregnant women were included in the study. Groups were formed: pregnant women with periodontal pathology entered the clinical group, in this group two subgroups were distinguished depending on adherence to treatment: with high adherence and low adherence to treatment. Periodontal diseases were structured according to the nomenclature and classification of periodontal diseases, adopted at a meeting of the presidium of the section of periodontics of the Russian Academy of Dentistry in 2001. To objectify the periodontal status in patients, the value of special indices was determined: the index of hygiene of the oral cavity according to Green-Vermillion (OHI-S), the Müllmanna bleeding index, the papillary-marginal-alveolar index (PMA) for assessing the presence, degree and depth of the inflammatory process in the gum. Statistical processing of the results of the work was carried out using the Statistica program (StatSoft, USA).

\section{The Results of the Study and Their Discussion}

Of the 398 examined pregnant women, 274 (68.8\%) had a periodontium pathology. In the structure of periodontal diseases, hypertrophic gingivitis (69\%) of light (37.2\%) and medium (25.2\%) prevalence was prevalent in frequency. In $17.1 \%$ of pregnant women, periodontitis, predominantly localized to mild severity, was defined. Chronic catarrhal gingivitis in all pregnant women was generalized, and ulcerative gingivitis was predominantly localized to $78.6 \%$.
The prevalence of signs of periodontal tissue damage was as follows: gingival hemorrhage $79.2 \%$, increased sensitivity of the teeth to chemical, thermal and mechanical stimuli $77 \%(n=21$, tartar $22.6 \%(n=62)$, periodontal pocket up to $4 \mathrm{~mm}-10.2 \%(\mathrm{n}=28)$, periodontal pocket $4-5 \mathrm{~mm}-6.9 \%(\mathrm{n}=19)$. The average number of sectarians with signs of periodontal tissue damage was: with bleeding gums -2.37 , with dental stone -0.81 , with a periodontal pocket up to $4 \mathrm{~mm}-0.74,4-5 \mathrm{~mm}$ and more -0.03 .

All patients with periodontal disease were prescribed standard treatment: professional oral hygiene with elimination of microbial plaque and tartar, oral baths, oral irrigation, applications, medical dressings with antiseptic and anti-inflammatory drugs, heparin electrophoresis, phonophoresis or injections of proteases, glucose, massage, darsonvalization. 107 (39.1\%) of the patients of the 1st group underwent the entire prescribed course of treatment and up to 36 weeks of gestation were under the control of the dentist. 167 (60.9\%) of women in 2 groups discontinued treatment and were classified as patients with low adherence to treatment. The main reason for the low adherence to treatment was the prejudice of patients to treatment during the gestational period, the expectation of an independent resolution of the dental problem with the end of the gestational period. In two groups, a comparative analysis of the index assessment of periodontal conditions in the dynamics of observation was carried out. The first examination was carried out 
at 1 and 2 trimesters of pregnancy, the second examination was carried out in the 3rd trimester before childbirth.

In group 1, the hygienic index significantly decreased by $28.6 \%$ ( $p<0.05)$, which indicated an improvement in the hygienic state of the oral cavity. In the 2nd group, the hygienic index increased by $76 \%$ ( $p<0.001$ ) due to an increase in the amount of plaque, tartar. This multidirectional change in the hygienic index in the two groups led to a statistically significant difference $(\mathrm{p}<0.01)$ in the hygiene index at 32-36 weeks of gestation: in the 1 st group it was $2.02 \pm 0.1$ points, in the 2 group $-4,48 \pm 0,5$ points. In the 1 st group, due to the active implementation of treatment measures, the PMA index decreased by 48. ( $\mathrm{p}<0.001)$, in the 2 nd group, almost doubled ( $\mathrm{p}$ $<0.001$ ) due to lack of treatment.

The lack of adherence to treatment was associated with an increase in inflammation of soft gingival tissue. In Group 1 patients, the gum bleeding index decreased by $35.1 \%$ ( $p<0.01$ ), and in patients of the 2 nd group did not change.

Thus, if during the first examination the condition of periodontal disease in pregnant women of the two groups did not differ, then in the dynamics of follow-up, adherence to the implementation of the full range of therapeutic measures was the key point, providing a favorable trend. Accounting for adherence to treatment is necessary, in order to correctly assess the factors contributing to the development of periodontal pathology.

\section{Conclusion}

a) The number of pregnant women with a low adherence to treatment is $60.9 \%$ higher than the number of patients who received a full course of medical treatment for periodontal pathology $39.1 \%$.

b) Adherence of pregnant women to the treatment of periodontal diseases is a key factor in ensuring favorable dynamics of periodontal conditions.

\section{References}

1. Arseenkova, O Yu TP Vasilyeva, AC Chumakov, AD Kuritsyn, V M Kuksenko, et al. (2010) Medico-social and psychological potential of pregnant women as consumers of dental health services. Bulletin of the Scientific Research Institute of Public Health 2: 16-20.

2. M Vogt, AW Sallum, JG Cecatti, SS Morais (2012) Factors associated with the prevalence of periodontal disease in low-risk pregnant women . Reproductive Health 9(3): 1-8.

3. Laine M A (2002) Effect of pregnancy on periodontal and dental health. Acta Odontol Scand 60(5): 57-64.

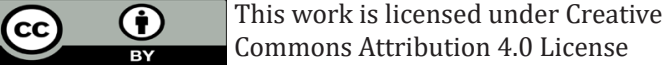

To Submit Your Article Click Here: Submit Article

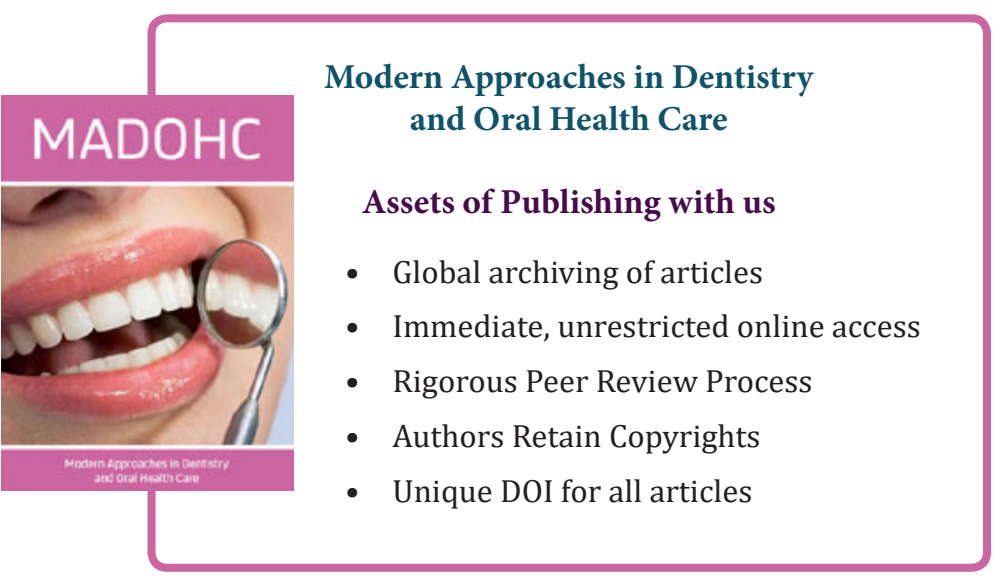

